\title{
Gamma Knife radiosurgery for recurrent or residual seizures after anterior temporal lobectomy in mesial temporal lobe epilepsy patients with hippocampal sclerosis: long-term follow-up results of more than 4 years
}

\author{
Eun Mi Lee, MD, PhD, ${ }^{1}$ Joong Koo Kang, MD, PhD, ${ }^{2}$ Sang Joon Kim, MD, PhD, ${ }^{3}$ \\ Seok Ho Hong, MD, PhD, ${ }^{4}$ Tae Sung Ko, MD, PhD, ${ }^{5}$ Sang Ahm Lee, MD, PhD, ${ }^{2}$ \\ Do Heui Lee, PhD, ${ }^{6}$ and Jung Kyo Lee, MD, PhD ${ }^{4}$
}

\begin{abstract}
1Department of Neurology, University of Ulsan College of Medicine, Ulsan University Hospital, Ulsan; and Departments of ${ }^{2}$ Neurology, ${ }^{3}$ Radiology, ${ }^{4}$ Neurosurgery, ${ }^{5}$ Pediatrics, and ${ }^{6}$ Medical Physics Support Center, University of Ulsan College of Medicine, Asan Medical Center, Seoul, Korea
\end{abstract}

\begin{abstract}
OBJECT Gamma Knife radiosurgery (GKRS) has proven efficacy in the treatment of drug-resistant mesial temporal lobe epilepsy with hippocampal sclerosis (MTLE-HS) and is comparable to conventional resective surgery. It may be effective as an alternative treatment to reoperation after failed temporal lobe surgery in patients with MTLE-HS. The purpose of this study was to investigate the efficacy of GKRS in patients with unilateral MTLE-HS who did not achieve seizure control or had recurrent seizures after anterior temporal lobectomy (ATL).

METHODS Twelve patients (8 males; mean age $35.50 \pm 9.90$ years) with MTLE-HS who underwent GKRS after failed ATL (Engel Classes III-IV) were included. GKRS targets included the remnant tissue or adjacent regions of the previously performed ATL with a marginal dose of 24-25 Gy at the $50 \%$ isodose line in all patients. Final seizure outcome was assessed using Engel's modified criteria during the final 2 years preceding data analysis. A comparison between signal changes on follow-up MRI and clinical outcome was performed.
\end{abstract}

RESULTS All patients were followed up for at least 4 years with a mean duration of $6.18 \pm 1.77$ years (range 4-8.8 years) after GKRS. At the final assessment, 6 of 12 patients were classified as seizure free (Engel Class la, $n=3 ; \mathrm{lc}, \mathrm{n}=$ 2; and Id, $n=1$ ) and 6 patients were classified as not seizure free (Engel Class II, $n=1 ; I I I, n=2$; and IV, $n=3$ ). Neither initial nor late MRI signal changes after GKRS statistically correlated with surgical outcome. Clinical seizure outcome did not differ significantly with initial or late MRI changes after GKRS.

CONCLUSIONS GKRS can be considered an alternative option when the patients with MTLE-HS who had recurrent or residual seizures after ATL refuse a second operation.

http://thejns.org/doi/abs/10.3171/2014.12.JNS141280

KEY WORDS Gamma Knife radiosurgery; mesial temporal lobe epilepsy; anterior temporal lobectomy; surgical failure; stereotactic radiosurgery

$\mathrm{M}$ ESIAL temporal lobe epilepsy (TLE) with hippocampal sclerosis (MTLE-HS) is one of the most important surgically amenable epilepsies for medically intractable epilepsy patients. ${ }^{19,23}$ Long-term seizure freedom after temporal lobe resection has been reported to be approximately $65 \% .^{8,21}$ Despite significant advances in the surgical procedure and the identification of surgical candidates, approximately $20 \%-30 \%$ of TLE patients continue to have seizures after conventional surgery. Moreover, the probability of seizure freedom 10 years after TLE surgery is reported to range between $40 \%$ and $50 \% .^{11}$

Several explanations have been proffered for these surgical failures, including incomplete resection of epileptogenic structures, generation or reactivation of new epileptogenic foci, a dual pathology, or, possibly, surgical

ABBREVIATIONS AED = antiepileptic drug; ATL = anterior temporal lobectomy; $A T L-A H=A T L$ with amygdalohippocampectomy; EEG = electroencephalography; GKRS = Gamma Knife radiosurgery; MTLE = mesial temporal lobe epilepsy; MTLE-HS = MTLE hippocampal sclerosis; TLE = temporal lobe epilepsy.

SUBMITTED June 9, 2014. ACCEPTED December 18, 2014.

INCLUDE WHEN CITING Published online July 10, 2015; DOI: 10.3171/2014.12.JNS141280.

DISCLOSURE The authors report no conflict of interest concerning the materials or methods used in this study or the findings specified in this paper. They confirm that they have read the Journal's position on issues involved in ethical publication and affirm that this report is consistent with those guidelines. 
scarring. ${ }^{22}$ Of these causes, incomplete resection of mesial structures is a potent and major cause of surgical failure. Although reoperation may be beneficial in highly selected patients with MTLE-HS, several reports have argued that $52 \%-63 \%$ of patients could be seizure free after reoperation (extension of the cortical resection along the surgical scar) for TLE. 1,7,17,22,24 Sometimes, additional invasive intracranial electroencephalography (EEG) recordings are needed to reevaluate and confirm the ictal onset zone before reoperation. Therefore, the potential for surgical morbidity and technical difficulties due to adhesion of neurovascular structures to adjacent surgical scar tissue is a concern for neurosurgeons.

As a noninvasive alternative approach to invasive resection, Gamma Knife radiosurgery (GKRS) has a proven efficacy in the treatment of drug-resistant MTLE with a seizure-free rate of $60 \%-67 \%$ at a greater than 24-month follow-up, which is comparable to the rate reported for conventional resective surgery. $2,3,14$ The advantages of GKRS include a decreased postprocedural morbidity, improved accessibility to deep structures, avoidance of open craniotomy, and reduced hospital stay. ${ }^{3}$ Thus, GKRS may be considered an alternative treatment to open cranial reoperation in patients with MTLE-HS who had recurrent or residual seizures after anterior temporal lobectomy (ATL). ${ }^{25}$ Because of the relatively small target volume of residual epileptic tissue, radiation can be safer than conventional GKRS as a primary treatment. However, there have been few reports regarding surgical outcomes of GKRS in homogeneous MTLE-HS groups of patients who underwent GKRS due to recurrent or residual seizure after temporal lobectomy.

In the present study, we investigated the characteristics and seizure outcomes following GKRS in patients with MTLE-HS who did not obtain seizure control or had recurrent seizures after previous surgery. We report the long-term follow-up results of GKRS in patients with an incomplete effect of ATL with amygdalohippocampectomy (ATL-AH).

\section{Methods \\ Patients' Characteristics Prior to GKRS}

We reviewed our database in the Epilepsy Center at the Asan Medical Center, Seoul, Korea, from 1995 to 2009. Five hundred thirty-four patients underwent resective surgery for medically intractable partial onset epilepsy at our institution. Of these, 425 patients underwent temporal lobe resections, including 330 patients with the histological diagnosis of MTLE. After ATL-AH in patients with MTLEHS, 229 patients $(76.3 \%)$ were seizure free (Engel Class I) at least 2 years before observation point. We included epileptic patients who fulfilled the following 4 criteria: 1) patients with MTLE-HS who underwent ATL-AH; 2) patients with unfavorable outcome (Engel Classes III and IV) after ATL-AH; 3) patients with MTLE-HS who were reevaluated for presurgical workup for residual or recurring seizures after ATL-AH; and 4) patients with MTLEHS who underwent GKRS for the treatment of the remaining seizures after ATL-AH. We included 12 patients with MTLE-HS who underwent GKRS after ATL. The institu- tional review board of Asan Medical Center approved this retrospective case series.

\section{Demographic Characteristics Before ATL}

Before the initial ATL-AH, all patients had drug-resistant epilepsy and received a comprehensive presurgical evaluation that included brain MRI, scalp video-EEG monitoring, and FDG-PET or ictal SPECT. Brain MRI studies with oblique coronal slices showed definite unilateral atrophy and/or increased signal intensities on T2-weighted or FLAIR sequences in the hippocampus ipsilateral to the epileptogenic temporal lobe. Interictal FDG-PET showed decreased metabolism in the temporal area ipsilateral to the hippocampal atrophy. Ictal SPECT showed increased blood perfusion in the temporal area ipsilateral to the hippocampal atrophy. Finally, presurgical workup confirmed the epileptic foci as being unilateral MTLE, consistent with unilateral hippocampal atrophy, in all included patients. The results of the initial preoperative workup, as well as patient characteristics, are summarized in Table 1.

\section{Surgical Outcomes After ATL and Reevaluation for GKRS}

Standard ATL-AH was performed in all patients without further invasive study (left/right 5:7). The histological diagnosis of MTLE-HS was documented in all patients except one because adequate tissue was not obtained (en bloc resection of hippocampal tissue was not available). The pathological findings showed hippocampal sclerosis only $(n=6)$ and possible dual pathology (hippocampal sclerosis plus other microscopic abnormality: hippocampal sclerosis plus microdysgenesis $[\mathrm{n}=2]$, hippocampal sclerosis plus mild cortical dysplasia $[\mathrm{n}=2]$, and hippocampal sclerosis plus subpial gliosis $[n=1])$. Postoperative MRI scans were reviewed by a neuroradiologist (S.J.K.) and confirmed a complete ATL, including resection of mesial structures (amygdala, uncus, head and body of the hippocampus, anterior parahippocampal gyrus, and entorhinal cortex), in 5 of 12 patients. Postoperative MRI showed a remnant hippocampal tail in 5 patients (41.7\%), remnant hippocampal tail and amygdala in 1 patient (8.3\%), and remnant amygdala in 1 patient $(8.3 \%)$.

After ATL-AH, 4 of the 12 patients were seizure free 1 year or more, whereas the remaining 8 patients had continuous seizures after ATL-AH. Of the 4 patients who were seizure free for more than 1 year, 2 were seizure free without antiepileptic drugs (AEDs) for 2.8 years and 13.3 years. Nevertheless, frequent seizures recurred after a considerable seizure-free period. All patients in the present study had a poor surgical outcome of Engel Classes III-IV after ATL-AH with a long-term follow-up of $5.9 \pm$ 4.36 years (range 1.3-14.1 years). All patients underwent a presurgical workup to reevaluate them for reoperation, including scalp video-EEG monitoring and brain MRI with oblique coronal images. If the results of video-EEG monitoring were considered congruent to the ipsilateral temporal lobe structure, which had been operated before, the suspected epileptogenic zone was the remnant or adjacent ipsilateral temporal lobe considering reoperation. However, if the EEG results were inconclusive or incongruent corresponding to ipsilateral temporal lobe origin, exploratory craniotomy for invasive monitoring was delib- 


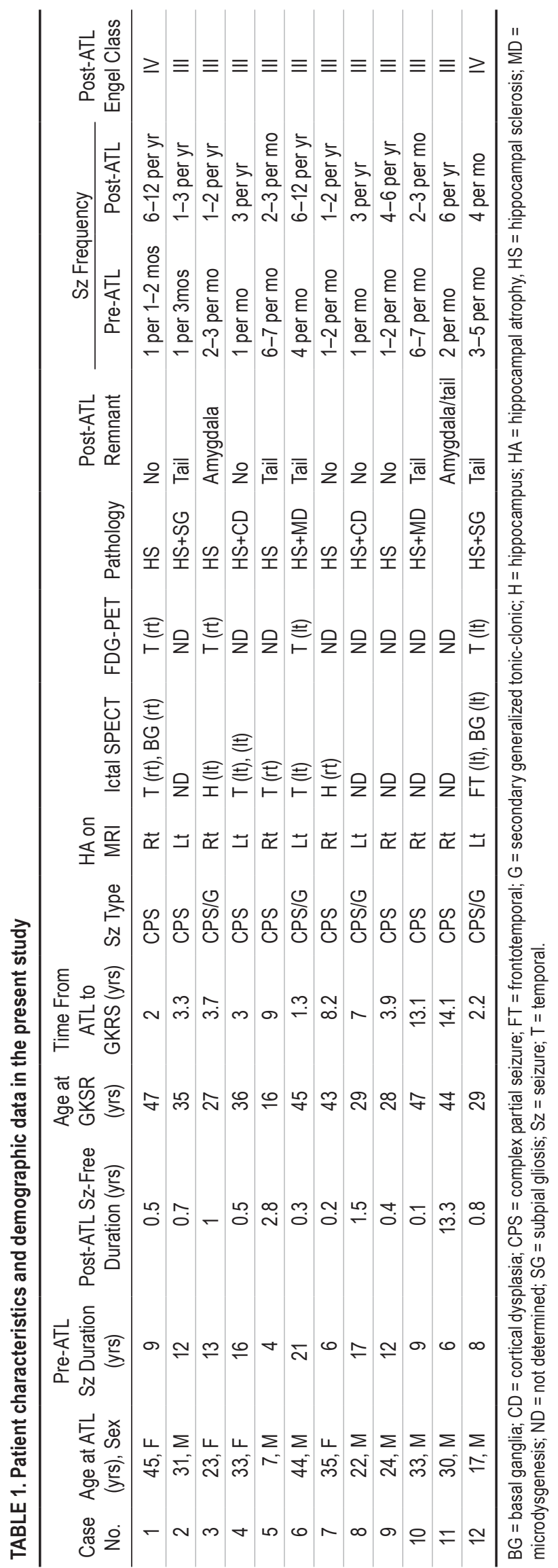

erated. GKRS was performed for patients who were willing to control the remaining seizures arising from adjacent structures after ATL, but refused to undergo reoperation.

\section{GKRS Protocol}

Radiosurgery was performed using a 201-source cobalt-60 model B or C Gamma Knife unit (Elekta Instruments). The stereotactic head frame was applied to the patients after administration of a local anesthetic agent to facilitate pain-free mounting. MRI using a 1-mm axial slice interval with no gap was performed for target localization. The senior author (J.K.L.) and technical assistant (D.H.L.) conducted treatment planning using GammaPlan software (Elekta Instruments). The target of the GKRS included any remnant structures following previously performed ATL-AH, or adjacent regions, such as the posterior fusiform gyrus, posterior parahippocampal gyrus, superior border of the amygdaloid complex, and tail of hippocampus, in cases of complete resection and depending on experimental decision. A representative planning MRI scan is illustrated in Fig. 1. The mean calculated volume at the $50 \%$ isodose target was $4183 \pm 1784.7 \mathrm{~mm}^{3}$ (range 1800-6900 $\mathrm{mm}^{3}$ ). A marginal dose of 24-25 Gy and mean number of isocenter shots of $7.8 \pm 5.7$ was administered to the target. The GKRS details are summarized in Table 2.

\section{Evaluation of Seizure Outcomes After GKRS}

Patients were regularly evaluated by investigators (J.K.K., S.A.L., and T.S.K.) at an outpatient clinic every 3 months after GKRS. Patients who had recurrent seizures sometimes visited more frequently. Patients were asked to maintain a diary of dates and details of seizures for presentation at every visit. Pre- and post-GKRS seizure reduction changes were calculated at the individual patient level. As the effectiveness of seizure reduction surgery is evident immediately after lobectomy but is usually delayed after GKRS, the final seizure outcome was assessed during the final 2 years before data analysis, and a minimum of 2 years of stable status was required for the final outcome classification. ${ }^{3}$ Post-GKRS seizure outcome was assessed according to Engel's modified classification using all 12 subclasses as follows ${ }^{3,5,6,20}$ : Class I, seizure free (Ia, completely seizure free since surgery; Ib, auras only since surgery; Ic, some seizures after surgery but seizure free for $\geq$ 2 years; and Id, atypical generalized convulsions following AED withdrawal only); Class II, rare seizures, with a maximum of 3 seizures per year (IIa, initially seizure free but currently has rare seizures; IIb, rare seizures since surgery; IIc, rare seizures for $\geq 2$ years; and IId, nocturnal seizures only, causing no disability); Class III, worthwhile improvement with a $>90 \%$ reduction of seizures (IIIa, worthwhile seizure reduction; and IIIb, prolonged seizure-free intervals for more than half of the follow-up but not $<2$ years); and Class IV, no worthwhile improvement $(<90 \%$ reduction in seizure frequency; IVa, 60-90\% reduction; IVb, no appreciable difference $[<60 \%$ reduction]). For categorical comparisons, this classification was divided into favorable (Engel Classes I and II) and unfavorable (Engel Classes III and IV) seizure outcomes. Anticonvulsant medications were modified (continued, increased, reduced, added to, and/or changed) by epileptologists (J.K.K., S.A.L., and 

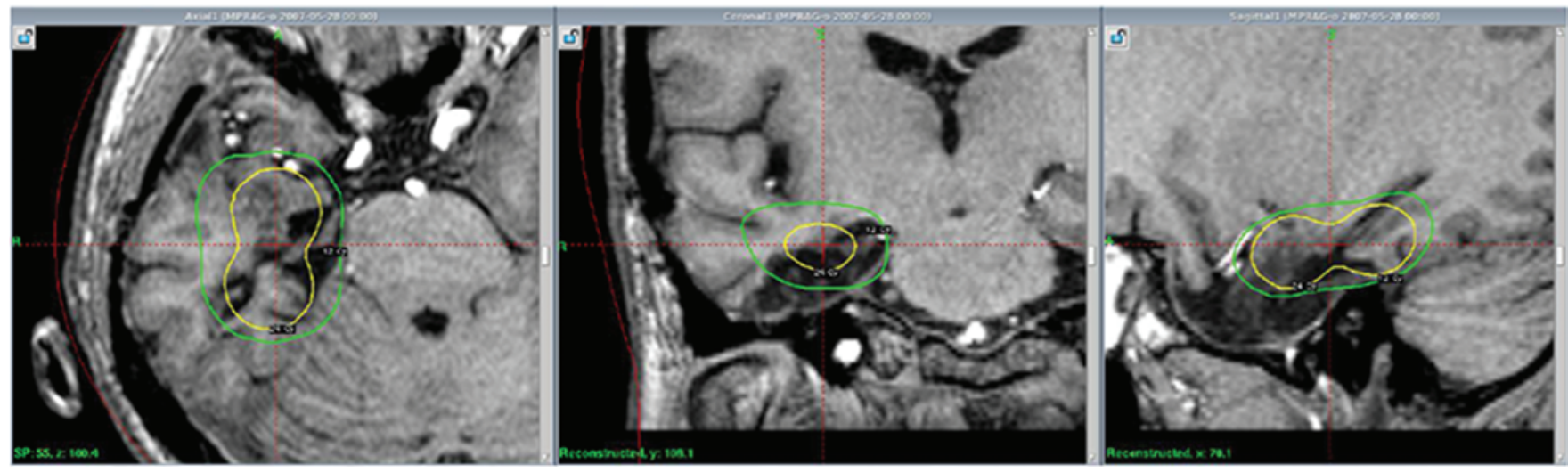

FIG. 1. Case 3. Representative screen capture of radiosurgical dose planning with previous right temporal lobectomy (axial, coronal, and sagittal slices). Fifty percent isodose lines of a total treatment of $24 \mathrm{~Gy}$ (yellow lines) delineated the treatment volume that contains the remnant hippocampal body and tail and adjacent regions. Green lines indicate the 12-Gy 50\% isodose lines used to limit radiation exposure to the brainstem. Figure is available in color online only.

T.S.K.). Formal visual field and neurophysiological tests were not performed in this study.

\section{Evaluation of MRI Changes After GKRS}

Follow-up MRI was performed in 10 of 12 patients to detect any radiation-induced signal changes at the target structures. Initial brain MRI was performed at 1-2 years in 9 patients after GKRS, and late follow-up MRI was performed at 4-8 years after GKRS in 8 patients. Thus, both initial and late follow-up MRI after GKRS was performed in 7 of 12 patients. All follow-up images were reviewed, and we discussed the post-GKRS signal changes. Signal changes were evaluated on each coronal $\mathrm{T} 2$-weighted and axial FLAIR and/or T1-weighted images. Two patients (Cases 1 and 5) underwent neither early nor late follow-up MRI after GKRS.

\section{Statistical Analysis}

Demographic data are reported with the use of range and median values for continuous variables. Some numeric variables are provided as the mean $\pm \mathrm{SD}$. The correlation between the clinical outcomes after GKRS and MRI changes or radiation parameters was analyzed. Categorical variables were analyzed using the Fisher's exact test, and continuous variables were analyzed with a MannWhitney U-test for unpaired variables. A p value $<0.05$

TABLE 2. Radiosurgical parameters and post-GKS seizure outcome

\begin{tabular}{|c|c|c|c|}
\hline Case No. & Target Vol $\left(\mathrm{mm}^{3}\right)$ & Radiation Dose (Gy) (50\% isodose) & No. of Target Points \\
\hline \multicolumn{4}{|l|}{ Favorable GKRS outcome } \\
\hline $1^{*}$ & 6300 & 24 & 2 \\
\hline $2^{*}$ & 6200 & 24 & 3 \\
\hline $3^{*}$ & 1800 & 24 & 6 \\
\hline $4 \dagger$ & 3600 & 24 & 22 \\
\hline $5 \dagger$ & 3600 & 24 & 9 \\
\hline $6 \dagger$ & 2500 & 24 & 3 \\
\hline $7 \dagger$ & 4700 & 24 & 11 \\
\hline Mean \pm SD & $4100 \pm 1730$ & & $8.0 \pm 7.0$ \\
\hline \multicolumn{4}{|l|}{ Unfavorable GKRS outcome } \\
\hline 8 & 2400 & 24 & 7 \\
\hline 9 & 2000 & 24 & 11 \\
\hline 10 & 5000 & 25 & 4 \\
\hline 11 & 5200 & 24 & 12 \\
\hline 12 & 6900 & 24 & 4 \\
\hline Mean \pm SD & $4300 \pm 2059$ & & $7.6 \pm 3.8$ \\
\hline Overall mean \pm SD & $4183 \pm 1785$ & $24.1 \pm 0.3$ & $7.8 \pm 5.7$ \\
\hline$p$ value (favorable vs unfavorable) & 0.88 & & 0.53 \\
\hline
\end{tabular}

\footnotetext{
* Early response.
}

$\dagger$ Delayed response. 
was considered statistically significant. All statistical calculations were performed using commercially available software (version 11.0.1, SPSS, Inc.).

\section{Results}

\section{Long-Term Clinical Effects}

The 12 patients had a mean age of $35.5 \pm 9.89$ years (range 16-47 years) at the time of the GKRS procedure; there were 8 males. The mean age at the time of ATL was $28.7 \pm 10.83$ years (range 7-45 years) and the median interval between the initial ATL and the GKRS was $5.9 \pm 4.36$ years (range 1.3-14.1 years). Five patients were treated on the left side and 7 were treated on the right. All patients were followed up for at least 4 years with a mean duration of $6.18 \pm 1.77$ years (range $4-8.8$ years) after GKRS. Eight of the patients had a longer than 5-year follow-up. The main characteristics of the patients before GKRS are summarized in Table 1.

At the final 2-year follow-up preceding this analysis, 6 of the 12 patients were considered seizure free (Engel Class Ia, $\mathrm{n}=3$; Ic, $\mathrm{n}=2$; Id, $\mathrm{n}=1$ ). One patient was in Class II, 2 were in Class III, and the 3 remaining patients were in Class IV. Table 3 and Fig. 2 depict the results obtained during the post-GKRS follow-up.
Among 6 seizure-free patients, 3 (Cases 1,2, and 3) were immediately seizure free after GKRS and never experienced a seizure (Engel Class Ia) during a mean follow-up period of 5.27 years (range 4.6-5.9 years). These patients were classified as the early response group. The patient in Case 6 reported just 1 episode of seizure following abrupt AED withdrawal after alcohol intake with 6.6 years of follow-up after GKRS (Engel Class Id). Two patients (Cases 4 and 5) initially did not show an improvement in seizure frequency for a couple of years after GKRS, but eventually had been seizure free for 4 years at the final outcome assessment (Engel Class Ic), and were classified as the delayed response group. One patient (Case 7) had no seizure reduction for 3 years after GKRS, but had a delayed response and had been seizure free for 2 years at the final follow-up (Engel Class II). Two patients (Cases 8 and 9) had no seizure reduction after GKRS with mean follow-ups of 5.3 and 8.8 years, but recently achieved 1 seizure-free year and were classified as Class IIIb. Thus, 7 patients achieved favorable outcomes (Engel Classes I and II) and the remaining 5 patients showed unfavorable outcomes (Engel Classes III and IV) after GKRS. No patient complained of any aggravation of memory problems, although pre- and post-GKRS neuropsychological test results were unavailable.

TABLE 3. Comparison of seizure outcome between pre- and post-GKRS and post-GKS MRI changes

\begin{tabular}{|c|c|c|c|c|c|c|c|c|}
\hline \multirow[b]{2}{*}{ Case No. } & \multicolumn{2}{|c|}{ Engel Class } & \multirow{2}{*}{$\begin{array}{l}\text { FU Duration } \\
\text { Post-GKRS (yrs) }\end{array}$} & \multicolumn{2}{|c|}{$\mathrm{AED}^{*}$} & \multirow[b]{2}{*}{ AED Change } & \multicolumn{2}{|c|}{ MRI Change* } \\
\hline & Pre-GKS & Post-GKS & & Pre-GKS & Post-GKS & & Early & Late \\
\hline \multicolumn{9}{|l|}{ Favorable GKRS outcome } \\
\hline $1 \dagger$ & IV & la & 5.9 & $\begin{array}{l}\text { LTG200, TPM150, } \\
\text { LEV1000 }\end{array}$ & LTG200, LEV1000 & Reduction & Yes & NA \\
\hline $2 \dagger$ & III & la & 5.3 & OXC1200, VPA1000 & OXC1200 & Reduction & NA & NA \\
\hline $3 \dagger$ & III & la & 4.6 & CBZ600 & CBZ600 & No change & Yes & Yes \\
\hline $4 \dagger \ddagger$ & III & Ic & 8.8 & CBZ800, LTG100 & CBZ400, LEV1000 & Reduction & NA & NA \\
\hline $5 \ddagger$ & III & Ic & 8.6 & $\begin{array}{l}\text { CBZ600, TPM300, } \\
\text { LTG200, } \\
\text { VGB2000 }\end{array}$ & CBZ600, LTG200 & Reduction & No & Yes \\
\hline $6 \ddagger$ & III & Id & 6.6 & $\begin{array}{l}\text { CBZ800, TPM50, } \\
\text { VPA1600 }\end{array}$ & $\begin{array}{l}\text { CBZ800, TPM50, } \\
\text { VPA1000 }\end{array}$ & Reduction & No & Yes \\
\hline $7 \ddagger$ & III & II & 4.9 & OXC1200, ZNS200 & OXC1200, ZNS200 & No change & No & Yes \\
\hline \multicolumn{9}{|l|}{ Unfavorable GKRS outcome } \\
\hline 8 & III & Illb & 5.3 & OXC1800, VPA1000 & $\begin{array}{l}\text { OXC1800, VPA1000, } \\
\text { LEV1000 }\end{array}$ & Addition & No & Yes \\
\hline 9 & III & Illb & 8.8 & CBZ1200, VPA1200 & CBZ1200, VPA1200 & No change & Yes & Yes \\
\hline 10 & III & IV & 4.3 & $\begin{array}{l}\text { CBZ1200, VPA1200, } \\
\text { PGB600 }\end{array}$ & CBZ600, TPM200 & Reduction & No & NA \\
\hline 11 & III & IV & 4 & OXC900 & OXC1200, VPA600 & Addition & NA & Yes \\
\hline 12 & IV & IV & 7.1 & $\begin{array}{l}\text { TPM100, CBZ1000, } \\
\text { VPA600, } \\
\text { ZNS200 }\end{array}$ & $\begin{array}{l}\text { TPM150, CBZ1200, } \\
\text { LEV2500 }\end{array}$ & Changed & Yes & Yes \\
\hline $\begin{array}{l}\text { p value (favorable vs unfa- } \\
\text { vorable) }\end{array}$ & & & & & & & 0.64 & \\
\hline $\begin{array}{l}\mathrm{CBZ}=\text { carbamazepine; } \mathrm{LEV}=1 \\
\text { ate; } \mathrm{ZNS}=\text { zonisamide. } \\
{ }^{*} \text { Values after the AED abbrevi } \\
\dagger \text { Early response. } \\
\ddagger \text { Delayed response. }\end{array}$ & $\begin{array}{l}\text { racetam; L } \\
\text { ss represe }\end{array}$ & $\begin{array}{l}\text { G = lamotrigi } \\
\text { mg/day. }\end{array}$ & & & & & & \\
\hline
\end{tabular}




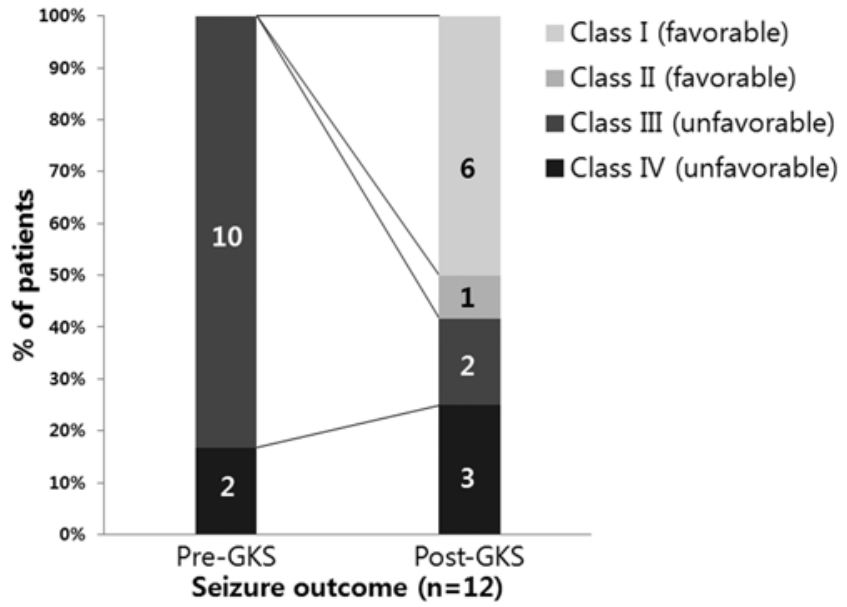

FIG. 2. Comparison of seizure outcome between pre-GKS and postGKS ( $n=12)$.

\section{Correlations Between Clinical Seizure Outcome and Radiation Parameters of GKRS}

There were no statistically significant differences in the target volume $\left(\mathrm{p}=0.88 ; 4100 \pm 1730 \mathrm{~mm}^{3}\right.$ vs $4300 \pm 2059$ $\left.\mathrm{mm}^{3}\right)$ and target points $(\mathrm{p}=0.53 ; 8.0 \pm 7.0$ vs $7.6 \pm 3.8)$ between patients who showed favorable and unfavorable post-GKRS outcomes (Table 2). In addition, neither target volume $\left(\mathrm{p}=0.82 ; 4000 \pm 1873 \mathrm{~mm}^{3}\right.$ vs $\left.4367 \pm 1849 \mathrm{~mm}^{3}\right)$ nor target points $(\mathrm{p}=0.31 ; 7.5 \pm 7.6$ vs $8.2 \pm 3.7)$ were different between patients who were immediately seizure free (Class Ia) after GKRS and those who were not.

\section{Appearance of MRI Signal Changes After GKRS}

Post-GKRS MRI changes, including focal T2 or FLAIR signal changes, were noted in 4 of 9 patients at the initial follow-up MRI and in all 8 patients at the late follow-up MRI after GKRS. Seven patients were studied with both initial and late follow-up MRI after GKRS. Three of these patients showed post-GKRS changes at initial MRI and subsequent extension of T2-weighted or FLAIR signal changes on late MRI (minimal in 1 patient and remarkable in 2 patients). The remaining 4 patients failed to show any changes on the initial follow-up MRI. However, all of these patients eventually demonstrated post-GKRS changes at the late follow-up MRI. Two patients (Engel Classes Ic and Ia) underwent neither initial nor late MRI. Figure 3 illustrates a typical MRI change after GKRS.

\section{Correlations Between Clinical Seizure Outcome and MRI Changes}

Comparisons between the MRI signal change at the initial and follow-up examinations and the clinical outcome were assessed. Post-GKRS outcomes were classified into Engel Classes I and II as the favorable outcome group and Engel Classes III and IV as the unfavorable outcome group. MRI signal changes were assessed by whether MRI signal change after GKRS was present or not on the initial and follow-up scans. Of 4 patients with initial MRI changes, 2 had favorable outcomes and 2 had unfavorable outcomes. Of 5 patients without initial MRI changes, 3 had favorable outcomes. A comparison of post-GKRS out-
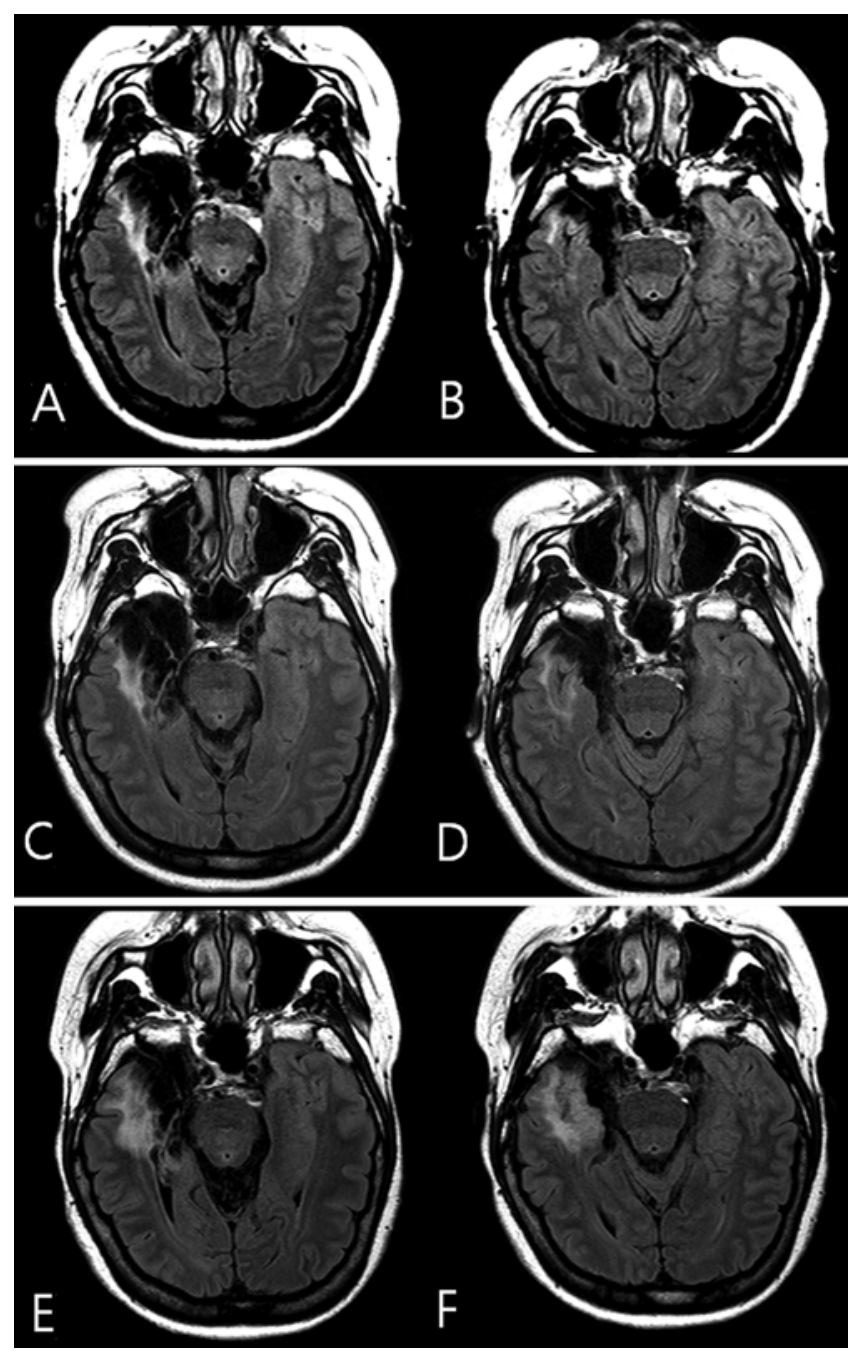

FIG. 3. Case 7. MRI scans demonstrating serial changes after GKRS. A and B: Axial FLAIR image obtained after ATL on the right, showing minimal residual posterior hippocampal tail. C and D: Axial FLAIR MRI obtained 1 year after GKRS, showing no significant change. E and F: Axial FLAIR image obtained 4 years after GKRS, showing remarkable extension of high signal adjunct on the right parahippocampal gyrus and temporal pole.

comes and initial MRI signal changes after GKRS showed no statistical differences $(p=0.64)$. All 8 patients with late follow-up MRI after GKRS had an MRI signal change. Among them, 4 patients had favorable outcomes but the remaining patients had unfavorable outcomes. Therefore, there were no significant differences in clinical seizure outcome according to the presence of initial or late MRI changes after GKRS.

\section{AEDs After GKRS}

All patients at the last follow-up were on a medication regiment. Among the 12 patients, the dose of AEDs was reduced in 6 patients, unchanged in 3 patients, and added to or changed to other AEDs in 3 patients. Five of the 6 patients with Engel Class I outcomes reduced their AED dose and the remaining patients continued the AEDs without change. Three of the 5 patients with unfavorable 
outcomes after GKRS added to or changed AEDs but the seizure frequency did not improve.

\section{Discussion}

The main finding of the present study is that GKRS produces favorable long-term surgical outcomes (Engel Classes I-II) after a mean follow-up of 6 years in $58.3 \%$ patients (7 of 12) with MTLE-HS who did not become seizure free or had recurrent seizures (Engel Classes III-IV) after ATL-AH. Reoperation with conventional surgery for the TLE remnant can obtain a seizure outcome rate ranging from $50 \%$ to $60 \%$. 1,7,17,22,24 However, considering the invasiveness and comparable surgical outcomes of the conventional reoperation in those who underwent a failed temporal lobectomy, our present results suggest that GKRS can be considered an alternative treatment to open cranial reoperation in patients with MTLE-HS who had residual or recurrent seizures after ATL-AH.

A noteworthy finding of our study is that immediate and persistent seizure-free statuses (Engel Subclass Ia) were noted in 3 of 12 patients (25\%) after GKRS. A high dose of 24 Gy with GKRS achieves early reductions in seizure frequency compared with a low dose of $20 \mathrm{~Gy}$ with GKRS, and the clinical effectiveness of the GKRS is delayed to 12 months after GKRS or is often preceded by a period of auras or seizure exacerbation. ${ }^{2,3}$ A recent study of GKRS for recurrent seizures after incomplete ATL reported that significant seizure reduction was found following the first 6 -month period after GKRS, although none of the patients became seizure free. ${ }^{25}$ In our current study, we used the previously documented effective treatment dose of 24 Gy to the residual tissue of the presumed epileptogenic zone and obtained an immediate response in 3 patients, compared with the relatively delayed effect of radiation in previous studies. We believe that this immediate response to GKRS may be better explained by neuromodulation effects rather than by tissue destruction.

On the other hand, some patients obtained a delayed response in becoming seizure free or only showed a significant seizure reduction in later periods. These patients (Cases 4 and 5) did not show definite signal changes on the initial MR image obtained 1 year after GKRS, unlike the significant signal changes on MRI performed 4 years or more after GKRS, suggesting that structural lesions rather than neuromodulating effects may be more important in seizure control. A previous study supports the belief that radiation-induced MRI changes correlate with the onset of seizure remission. ${ }^{4}$

Overall, our present findings indicated that the presence or absence of signal changes on MRI at 1-2 years after GKRS did not correlate with long-term surgical outcome in any group, including the immediate response group. In addition, all patients showed signal changes on MRI performed 4 years or later after GKRS, even though the extent of the signal changes on MRI did not correlate with the clinical outcome.

The mechanisms by which GKRS reduces or eliminates seizures are unclear. Radiosurgery has been shown to obtain seizure remission by significant injury to neuronal tissue in the presumed seizure focus. ${ }^{4}$ In contrast, the status of radiation-induced edema did not influence the seizure control of epilepsy associated with vascular malformations..$^{10}$ Furthermore, seizure remission after radiosurgery of other epileptic lesions, such as the hypothalamic hamartoma or vascular malformation, does not require radiological changes consistent with radionecrosis. ${ }^{15,18}$ Therefore, tissue destruction is insufficient to explain the clinical effects produced, suggesting function modulation while preserving basic processing. ${ }^{12,13}$ Our present findings also showed that seizure outcome after GKRS is not related to MRI changes, which is consistent with results showing that tissue necrosis is not always required for the antiepileptic effect.

Previous studies have reported that a low radiation dose $(<20 \mathrm{~Gy})$ is often associated with the failure of GKRS to control seizures and a lack of MRI changes ${ }^{9}$ and that a higher dose causes greater clinical effects and radiological changes. ${ }^{4}$ In our present study, we used the previously documented effective treatment dose of 24 Gy to the residual tissue of the presumed epileptogenic zone, although the target volume was lower than in previous studies (range $1.8-6.9 \mathrm{~cm}^{3}$ vs $5.5-9 \mathrm{~cm}^{3}$ ) that used GKRS as an initial surgical treatment in patients with MTLE-HS. ${ }^{2,14}$ Ablation of the entire area of the amygdala and hippocampus requires a large radiation dose and risks local radiation reaction and a greater radiation dose, which can lead to large brain edema and subsequent steroid treatment. Some patients in our current series experienced transient headache, but only one patient had significant brain edema and required steroid treatment. In this regard, the relatively small target volume in our study incorporating residual epileptic tissue may be safer than conventional GKRS as a primary treatment, which requires steroid treatment in up to $60 \%$ of patients. ${ }^{2}$

In comparison with the approximately 9- to 12-month delay in the appearance of the first neuroradiological changes described in previous reports, ${ }^{3,4,14,16}$ more than half of the patients in our present study showed absent or minimal radiological changes at 1 year after GKRS and had particularly delayed GKRS-related MRI changes 4 years later. It may be that the underlying extensive scar change on MRI after ATL could obscure newly developed radiation changes, making them almost indistinguishable from existing scar tissue.

With regard to conventional reoperation surgery, 5 of the 12 patients were not improved or were insufficiently improved after GKRS. In the present study, the epileptogenic zone was reconfirmed as the temporal lobe, ipsilateral to the previous operation site, on presurgical reevaluation that included video-EEG monitoring, MRI, and PET. Studies regarding failed epilepsy cases for MTLE-HS revealed the ipsilateral temporal lobe to be the most common cause of recurrent seizures. The causes of recurrent seizures include the generation of occult or new epileptogenic zones as well as residual epileptogenic tissue..22 Thus, the reasons for these failures in these patients are not well understood, although the failures are probably related to the extension of the epileptogenic zone beyond the limits of the radiosurgery. ${ }^{3}$ Another possible reason for the failure in these patients may be that conventional radiation doses (24 Gy) induce cellular changes, as seen on the MRI scan, but are insufficient to suppress seizures.

The present study has some limitations. First, we did 
not perform a follow-up visual field test or neuropsychological test. However, no patients in our series complained of memory decline or had noticeable visual field problems. Moreover, none of our patients in the present study had any permanent neurological morbidity or mortality after GKRS. Second, we did not perform follow-up MRI in 3 patients who showed good clinical outcomes (Engel Class I). However, we performed follow-up MRI in most of the patients, which did not affect the analysis of the results.

\section{Conclusions}

ATL is the most widely performed standard treatment for MTLE-HS. However, the optimal treatment for failed epileptic surgery in TLE is not currently established, and the question remains as to whether the exact epileptogenic focus related to relapse still matters in spite of extensive reevaluation using integration of multimodal approaches. GKRS at residual tissue after sufficient reinvestigation has no major safety concerns, coupled with the advantage of a relatively small dose of radiation. Comparable seizure relief compared with open cranial reoperation indicates that GKRS can be considered as an alternative noninvasive treatment in patients with MTLE-HS who have residual or recurrent seizures after ATL but refuse reoperation. Our present findings can contribute to decision making when treating patients with failed surgery for MTLE-HS.

\section{References}

1. Awad IA, Nayel MH, Lüders H: Second operation after the failure of previous resection for epilepsy. Neurosurgery 28:510-518, 1991

2. Barbaro NM, Quigg M, Broshek DK, Ward MM, Lamborn KR, Laxer KD, et al: A multicenter, prospective pilot study of gamma knife radiosurgery for mesial temporal lobe epilepsy: seizure response, adverse events, and verbal memory. Ann Neurol 65:167-175, 2009

3. Bartolomei F, Hayashi M, Tamura M, Rey M, Fischer C, Chauvel P, et al: Long-term efficacy of gamma knife radiosurgery in mesial temporal lobe epilepsy. Neurology 70:1658-1663, 2008

4. Chang EF, Quigg M, Oh MC, Dillon WP, Ward MM, Laxer $\mathrm{KD}$, et al: Predictors of efficacy after stereotactic radiosurgery for medial temporal lobe epilepsy. Neurology 74:165172,2010

5. Costello DJ, Shields DC, Cash SS, Eskandar EN, Cosgrove GR, Cole AJ: Consideration of epilepsy surgery in adults should be independent of age. Clin Neurol Neurosurg 111:240-245, 2009

6. Engel J Jr, Van Ness P, Rasmussen TB, Ojemann LM: Outcome with respect to epileptic seizures, in Engel J Jr (ed): Surgical Treatment of the Epilepsies, ed 2. New York: Raven Press, 1993, pp 609-621

7. Germano IM, Poulin N, Olivier A: Reoperation for recurrent temporal lobe epilepsy. J Neurosurg 81:31-36, 1994

8. Jeong SW, Lee SK, Hong KS, Kim KK, Chung CK, Kim H: Prognostic factors for the surgery for mesial temporal lobe epilepsy: longitudinal analysis. Epilepsia 46:1273-1279, 2005

9. Kawai K, Suzuki I, Kurita H, Shin M, Arai N, Kirino T: Failure of low-dose radiosurgery to control temporal lobe epilepsy. J Neurosurg 95:883-887, 2001

10. Kurita H, Kawamoto S, Suzuki I, Sasaki T, Tago M, Terahara A, et al: Control of epilepsy associated with cerebral arteriovenous malformations after radiosurgery. J Neurol Neurosurg Psychiatry 65:648-655, 1998
11. McIntosh AM, Kalnins RM, Mitchell LA, Fabinyi GC, Briellmann RS, Berkovic SF: Temporal lobectomy: long-term seizure outcome, late recurrence and risks for seizure recurrence. Brain 127:2018-2030, 2004

12. Régis J: Radiosurgery as neuromodulation therapy! Acta Neurochir Suppl 116:121-126, 2013

13. Régis J, Carron R, Park M: Is radiosurgery a neuromodulation therapy? J Neurooncol 98:155-162, 2010

14. Régis J, Rey M, Bartolomei F, Vladyka V, Liscak R, Schröttner O, et al: Gamma knife surgery in mesial temporal lobe epilepsy: a prospective multicenter study. Epilepsia 45:504-515, 2004

15. Régis J, Scavarda D, Tamura M, Nagayi M, Villeneuve N, Bartolomei F, et al: Epilepsy related to hypothalamic hamartomas: surgical management with special reference to gamma knife surgery. Childs Nerv Syst 22:881-895, 2006

16. Régis J, Semah F, Bryan RN, Levrier O, Rey M, Samson Y, et al: Early and delayed MR and PET changes after selective temporomesial radiosurgery in mesial temporal lobe epilepsy. AJNR Am J Neuroradiol 20:213-216, 1999

17. Salanova V, Markand O, Worth R: Temporal lobe epilepsy: analysis of failures and the role of reoperation. Acta Neurol Scand 111:126-133, 2005

18. Schäuble B, Cascino GD, Pollock BE, Gorman DA, Weigand S, Cohen-Gadol AA, et al: Seizure outcomes after stereotactic radiosurgery for cerebral arteriovenous malformations. Neurology 63:683-687, 2004

19. Spencer SS, Berg AT, Vickrey BG, Sperling MR, Bazil CW, Shinnar S, et al: Initial outcomes in the Multicenter Study of Epilepsy Surgery. Neurology 61:1680-1685, 2003

20. Tanriverdi T, Olivier A, Poulin N, Andermann F, Dubeau F: Long-term seizure outcome after mesial temporal lobe epilepsy surgery: corticalamygdalohippocampectomy versus selective amygdalohippocampectomy. J Neurosurg 108:517524, 2008

21. Téllez-Zenteno JF, Dhar R, Wiebe S: Long-term seizure outcomes following epilepsy surgery: a systematic review and meta-analysis. Brain 128:1188-1198, 2005

22. Vale FL, Pollock G, Benbadis SR: Failed epilepsy surgery for mesial temporal lobe sclerosis: a review of the pathophysiology. Neurosurg Focus 32(3):E9, 2012

23. Wiebe S, Blume WT, Girvin JP, Eliasziw M: A randomized, controlled trial of surgery for temporal-lobe epilepsy. N Engl J Med 345:311-318, 2001

24. Wyler AR, Hermann BP, Richey ET: Results of reoperation for failed epilepsy surgery. J Neurosurg 71:815-819, 1989

25. Yen DJ, Chung WY, Shih YH, Chen C, Lirng JF, Yiu CH, et al: Gamma knife radiosurgery for the treatment of recurrent seizures after incomplete anterior temporal lobectomy. Seizure 18:511-514, 2009

\section{Author Contributions}

Conception and design: JK Lee, Kang. Acquisition of data: JK Lee, EM Lee, Kang, Hong, Ko, SA Lee. Analysis and interpretation of data: JK Lee, EM Lee, Kang. Drafting the article: EM Lee. Critically revising the article: JK Lee, EM Lee. Reviewed submitted version of manuscript: JK Lee, EM Lee. Approved the final version of the manuscript on behalf of all authors: JK Lee. Statistical analysis: EM Lee. Administrative/technical/material support: Kim, Hong, Ko, SA Lee, DH Lee. Study supervision: Kang.

\section{Correspondence}

Jung Kyo Lee, Department of Neurosurgery, University of Ulsan College of Medicine, Asan Medical Center, 88, Olympic-ro 43-gil, Songpa-gu, Seoul, 138-736, South Korea. email: jklee2@ icloud.com. 